\title{
Schlechter Schlaf im Klimakterium weist auf Arteriosklerose der Karotiden hin
}

\author{
Rund die Hälfte aller Frauen leidet in den Wechseljahren unter Schlafstörungen. Eine \\ Studie zeigt nun, dass mit diesem Phänomen auch Gefäßerkrankungen assoziiert sind.
}

\begin{abstract}
Schlafstörungen werden von viele Frauen als die belastendsten Wechseljahresbeschwerden geschildert. Als Ursache für die Symptome gelten sinkende Östrogenspiegel, aber auch Depressionen können eine Rolle spielen. Zudem ist bekannt, dass eine Schlafapnoe in den Wechseljahren häufiger auftritt. Das alles kann zu einer nachhaltigen Leistungsminderung am Tag führen.

In einer vom National Heart, Lung, and Blood Institute (NHLBI) aus den USA gesponserten Studie wurde nun gezeigt, dass bei Schlafstörungen im Klimakterium häufig Arterioskleroseprozesse in den Karotiden vorliegen. Dafür wurden 256 periund postmenopausale Frauen im Alter zwischen 40 und 60 Jahren mit noch vorhandenem Uterus und zumindest einem Ovar untersucht. Alle Teilnehmerinnen waren Nichtraucherinnen, keine von ihnen arbeitete Nachtschicht. Ausgeschlossen wurden anamnestische und aktuelle klinische Hinweise auf eine Herz-Kreislauf-Erkrankung sowie die Einnahme von Schlafmitteln, Betablockern, Kalziumantagonisten, ACEHemmern oder Insulin.
\end{abstract}

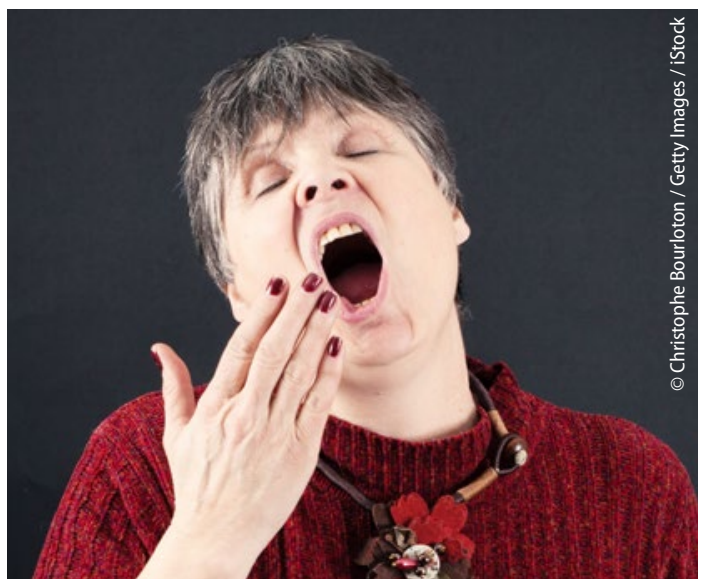

Ständig müde? Dann könnte auch eine Untersuchung der Halsschlagadern angeraten sein.

Alle Studienteilnehmerinnen trugen drei Tage lang einen Aktigrafen zum Schlafmonitoring. Hitzewallungen wurden mittels eines sternalen Sensors dokumentiert. Die Schlafqualität wurde mit dem Pittsburgh Sleep Quality Index (PSQI) erfasst. Mittels einer Duplexuntersuchung der Karotiden wurde die Intima-Media-Dicke bestimmt und außerdem der Plaques-Status evaluiert. Eine Blutabnahme diente der Bestimmung der Blutfett- und Blutzuckerwerte, ferner wurden die Insulin- und Östrogenspiegel festgehalten.
Die Ergebnisse zeigten, dass vermehrte Schlafstörungen und verkürzter Schlaf mit einer vermehrten Plaques-Bildung und einer $\mathrm{Zu-}$ nahme der Intima-Media-Dicke korrelierten (Odds Ratio 1,58). Eine Schlafdauer von über sieben Stunden war nicht protektiv. Auch eine generell schlechtere Schlafqualität korrelierte mit einer Zunahme arteriosklerotischer Veränderungen an den Halsschlagadern. Dies zeigte sich auch unter Berücksichtigung der Kovariaten wie Östrogenspiegel, Hitzewallungen und depressive Symptome.

- Thurston RS, Chang Y, von Känel R et al. Sleep characteristics and carotid atherosclerosis among midlife women. Sleep 2016, online 10. Dezember; pii:sp-00299-16

\section{KOMMENTAR}

Die Autoren fordern ein Herz- und Gefäßscreening bei Frauen mit Schlafstörungen in den Wechseljahren - auch wenn man festhalten muss, dass eine Kausalität durch diese Studie nicht bewiesen ist. Dass Schlafstörungen mit arteriosklerotischen Veränderungen an den Halsschlagadern Hand in Hand gehen, ist aber nicht abzustreiten.

Prof. Dr. med. C. Diehm

\section{Hier steht eine Anzeige.}

